\title{
Mission Performance as Point Performance
}

\author{
Robert A. McDonald* \\ California Polytechnic State University, San Luis Obispo, CA, 93407
}

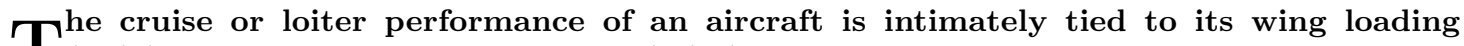
$\mathcal{I}(W / S)$ and its thrust to weight ratio $(T / W)$. Paradoxically, mission performance is often not considered when these fundamental aircraft parameters are determined in conceptual design.

In this research, the traditional constraint diagram is extended to include contours of range parameter. This point performance metric represents the mission performance capability of the aircraft without sizing the aircraft to a particular mission. This gives the designer an immediate and intuitive understanding of the tradeoff between the point and mission performance of the aircraft.

This improved constraint diagram presents the designer with a more complete basis for understanding and weighing the consequences of decisions. Through improved insight, the secondary impact of technologies can be better understood early in the design process.
\end{abstract}

\section{Introduction}

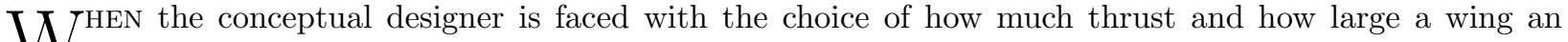
W aircraft should have, the $T / W$ vs. $W / S$ constraint diagram is often the tool of choice. Sometimes called the 'matching plot', the constraint diagram concisely represents an aircraft's ability to meet the specified point-performance requirements. The requirements typically represented include takeoff distance and/or balanced field length, landing distance, approach and/or stall speed, rate of climb, climb gradient, ceiling, dash, sustained and/or instantaneous turn, etc. ${ }^{1-3}$

Unfortunately, when used in this way, the constraint diagram gives no guidance as to the effect of thrust and wing loading on the mission performance of the aircraft. However, these parameters are crucial in determining the cruise or loiter efficiency of the aircraft. In fact, the fundamental tradeoffs between point and mission performance are completely overlooked by the conventional constraint diagram.

In order to investigate the effect of thrust and wing loading on mission performance, the designer is typically forced to delve deeper into the design process, sizing the vehicle to a design mission. ${ }^{4}$ Consequently, some practitioners have moved away from the constraint diagram entirely, preferring to skip directly to sizing a vehicle; this is often accomplished with a unified sizing and synthesis tool.

In this research, the traditional constraint diagram is extended to include contours of range parameter. This point performance metric represents the mission performance capability of the aircraft without sizing the aircraft to a particular mission. This gives the designer an immediate and intuitive understanding of the tradeoff between the point and mission performance of the aircraft.

\section{Example Aircraft}

An example aircraft will be employed to illustrate the concepts discussed in this paper. The example aircraft is defined in terms of a drag polar and an engine deck. Rather than use greatly simplified equations for the drag, thrust, and fuel consumption, tabulated data taken from a real-world aircraft was used. All of the equations and procedures developed in this work are applicable to an arbitrary aircraft model.

The drag of a DC-10 in cruise configuration was used for the example aircraft. Figures 1(a) and 1(b) depict the drag polar and drag rise behavior for a variety of lift coefficients for this aircraft. This drag data was digitized from data presented in the McDonnell Douglas performance course. ${ }^{5}$

*Lockheed Martin Assistant Professor, Aerospace Engineering, One Grand Avenue, AIAA Member. 


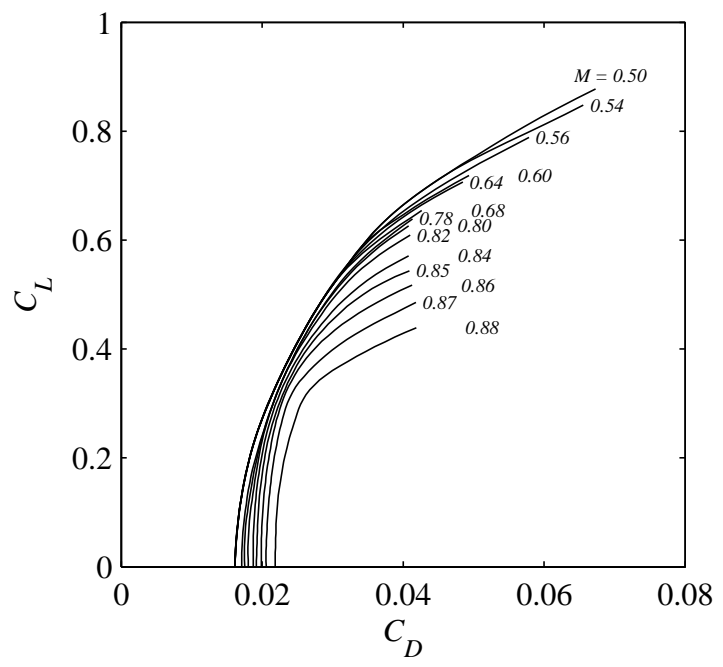

(a) Cruise drag polar.

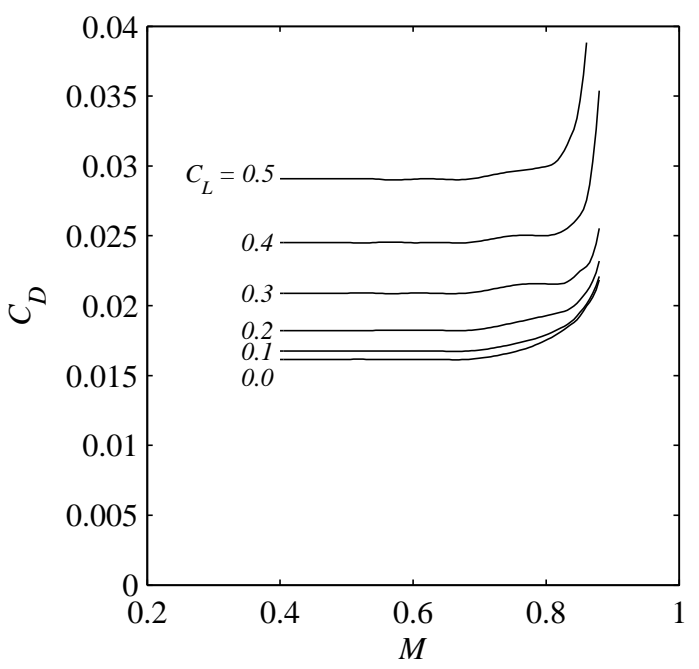

(b) Drag rise characteristics for selected lift coefficients.

Figure 1. DC-10 Cruise aerodynamics. ${ }^{5}$

The thrust and fuel consumption of a CF6-50 was used for the example aircraft. Figure 2(a) depicts the full throttle thrust lapse of the engine throughout the operational envelope. Figure 2(b) depicts the thrust specific fuel consumption (TSFC) of the engine at $80 \%$ thrust throughout the operational envelope. The part-power performance of the engine is exemplified by the thrust hook for operation at $M=0.8$ and $h=36,089 \mathrm{ft}$. given as Figure 2(c). This engine data was digitized from data presented in the CF6 Installation Manual. ${ }^{6}$

Construction of a constraint diagram relies on a 'rubber engine' assumption; this assumption represents the engine as a certain cycle which may be scaled up or down with $T / W$ as desired. This neglects any effects of scale on the engine cycle and presumes an engine company willing to build any engine to order. To 'rubberize' a given engine, the thrust is scaled by the sea level rated thrust. This thrust ratio, $\alpha$, represents both the thrust lapse due to speed and altitude, but also any reduction in thrust due to throttling.

Construction of a constraint diagram also relies on a 'rubber airframe' assumption; this represents the airframe as a drag polar held fixed throughout the study. For all-wing and some other advanced concepts, this assumption is quite good, only neglecting any Reynolds number scale effects on the aircraft drag. However, for transport aircraft with fixed payload requirements, conceptual design changes in wing loading are most often realized through changes in wing area; these changes can have significant effect on the drag polar for a configuration.

\section{Cruise Operating Point}

The aircraft designer often has limited ability to choose the operating conditions of the aircraft. Cruise speed and altitude may be stated as requirements from the prospective customer. Furthermore, a commercial aircraft will need to work within the existing air traffic control infrastructure, flying at appropriate flight levels and at speeds set by air traffic controllers to maintain safe separation limits. The designer's job is to tailor the aircraft to maximize performance at some specified condition whereas the pilot and performance engineer's job is to tailor the existing aircraft's operation to maximize performance. In this paper, the operating point as specified by cruise Mach and altitude are fixed at $M=0.8$ and $h=36,089 \mathrm{ft}$.

\section{Long Range Performance}

The Breguet range equation provides insight into the integrated mission performance of an aircraft without requiring the complexity of a complete mission model. When written as Equation 1, the crucial elements for long range flight are clearly evident. An aircraft designed for efficient cruse should have high 


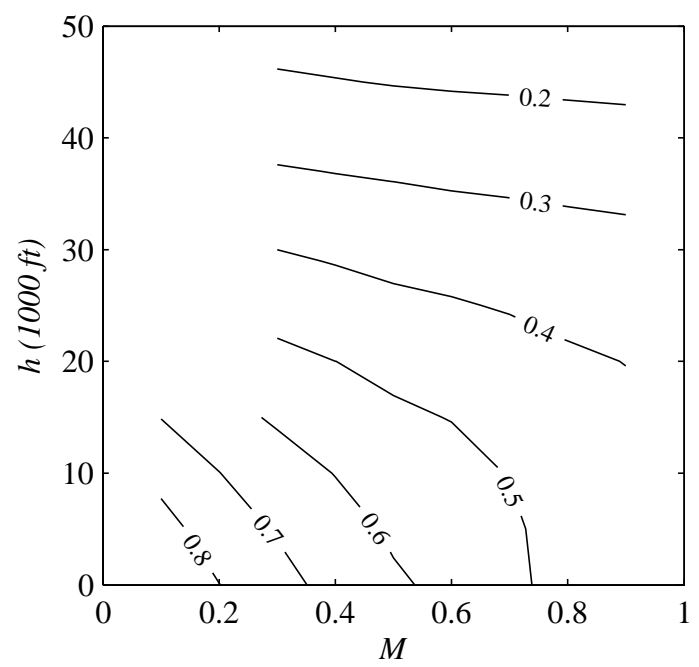

(a) Thrust lapse $(\alpha)$ at full power.

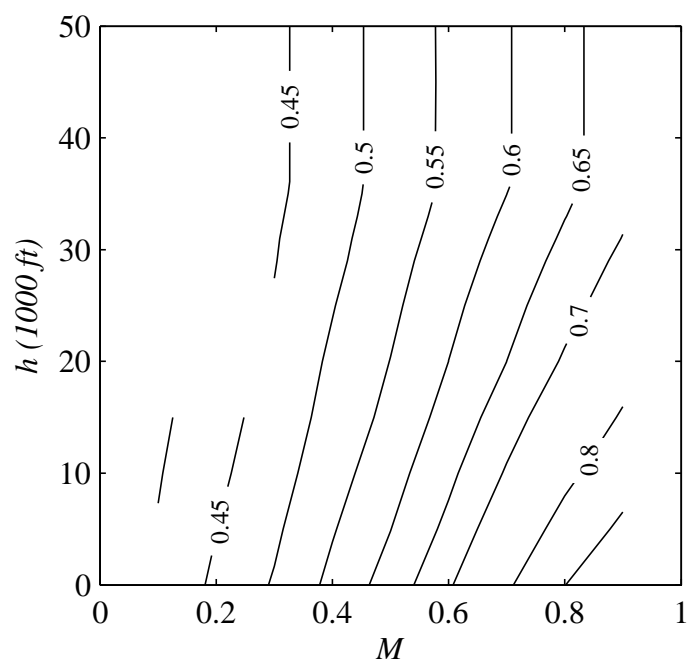

(b) TSFC behavior at $80 \%$ thrust.

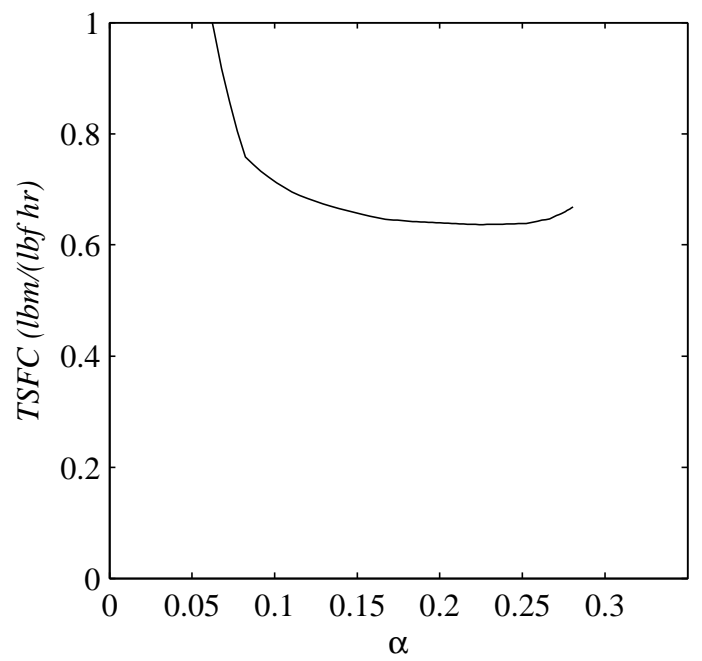

(c) Rubberized thrust hook at the Tropopause $M=0.8$.

Figure 2. CF6 Engine performance. 
aerodynamic efficiency $(V L / D)$, low thrust specific fuel consumption $(T S F C)$, and requires a large fuel load $\left(W_{f}\right)$ and a low empty weight $\left(W_{e}\right)$ for a given payload weight $\left(W_{p}\right)$.

$$
R=\frac{V}{T S F C} \frac{L}{D} \ln \left(1+\frac{W_{f}}{W_{p}+W_{e}}\right)
$$

The coefficients in the front of this equation can be grouped into quantities which are useful for further simplifying the range behavior of an aircraft. The specific range $S R$, defined in Equation 2, is typically expressed in units of nautical miles per thousand pounds of fuel. The specific range is directly analogous to fuel efficiency expressed as miles per gallon (MPG) for a family car. These metrics give an indication of a vehicle's efficiency, but do not indicate how much fuel is required for a particular mission (distance and payload). They also leave out the effects fuel fraction of the vehicle, but include some effect of the vehicle size.

$$
S R \equiv \frac{V}{T S F C D}
$$

Similarly, the range parameter $R P$, defined in Equation 3, is typically expressed in units of nautical miles. The range parameter abstracts the specific range by removing the effect of the vehicle scale. This makes it suitable for consideration at the earliest stages of design when the constraint diagram is used to guide decisions. However, the range parameter does not enjoy the intuitive interpretation of specific range.

$$
R P \equiv \frac{V}{T S F C} \frac{L}{D}
$$

In this study, we aim to treat range parameter as a point performance quantity which can be considered alongside traditional point performance constraints. The quantities which appear in each of these equations are sometimes grouped in order to simplify discussion and to provide intuition to the designer for how to effect changes in a vehicle's performance; at this stage, this is predominately accomplished through consideration of the thrust to weight ratio, $T / W$, and wing loading, $W / S$, of the aircraft. Although the abbreviated symbol $T / W$ is often used, the quantity being considered is the ratio of the sea level static rated thrust to the maximum takeoff gross weight of the aircraft, $T_{s l s, \max } / W_{T O}$. Similarly, the abbreviated symbol $W / S$ refers to the ratio of the maximum takeoff gross weight to the reference area $W_{T O} / S$.

\section{Aerodynamic Efficiency}

The effect of aerodynamic efficiency on aircraft range can be understood by considering the term $V L / D$, or often $M L / D$. These metrics can be calculated and plotted as contours across the operating envelope as in Figure 3.

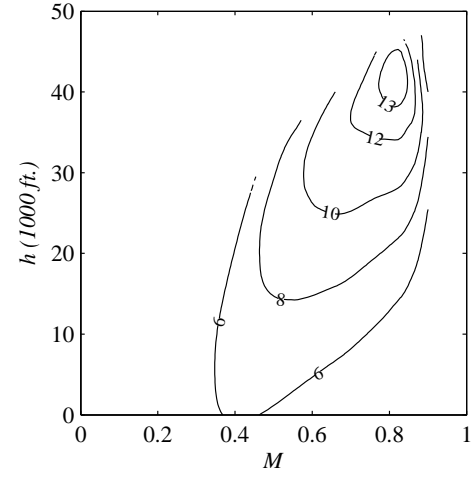

(a) $W / S=100 \mathrm{lbf} / \mathrm{ft}^{2}$.

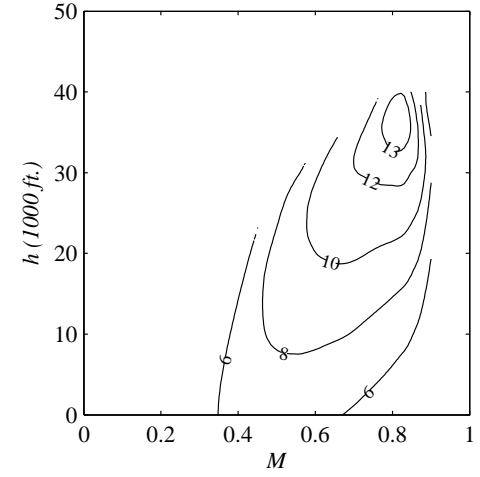

(b) $W / S=130 \mathrm{lbf} / \mathrm{ft}^{2}$.

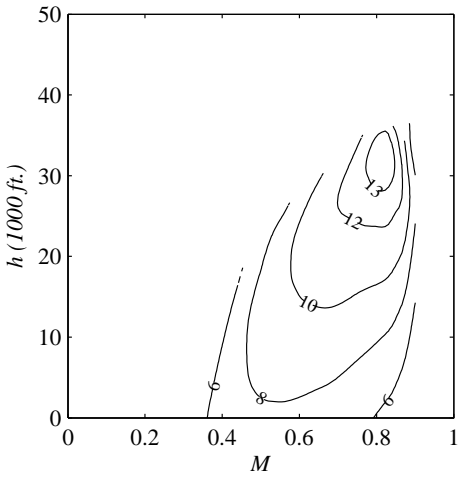

(c) $W / S=160 \mathrm{lbf} / \mathrm{ft}^{2}$.

Figure 3. Contours of $M L / D$ for three wing loadings and weight fraction $\beta=0.8$.

Figures 3 (a), (b), and (c) depict the cruise aerodynamic efficiency of three aircraft with differing wing loading, $W / S=100,130$, and $160 \mathrm{lbf} / \mathrm{ft}^{2}$, respectively. Increasing wing loading primarily serves to shift the 
contours down, reducing the altitude for maximum cruise aerodynamic efficiency. Consequently, wing loading can be viewed as a primary means for the designer to match the aircraft to the desired cruise altitude.

Generation of $M L / D$ contours requires knowledge or specification of the drag polar $\left(C_{D}=f\left(C_{L}, M, h\right)\right)$, the assumed atmosphere model $((\rho, a)=f(h))$, the wing loading $(W / S)$, and the weight fraction $\left(\beta \equiv W / W_{T O}\right)$ during cruise. For the designer to influence the cruise aerodynamic efficiency, they must exert influence on those quantities. The aircraft designer has no capacity to change the behavior of the earth's atmosphere. The cruise weight fraction is a representative average value which is a fallout of a detailed vehicle sizing process. This leaves the designer with some influence on the drag polar and the wing loading.

The designer can change the drag polar for the aircraft - many fundamental design decisions contribute to determine the drag polar. These changes can generally be thought of as reducing drag - cleaning up the aircraft to reduce parasite drag or improving the wing to reduce induced drag. The secondary effect of adjusting the balance between induced and parasite drag for an aircraft is also critical. However, due to the mission requirements and the fundamental physics driving the drag of an aircraft, the conceptual designer does not have a great deal of freedom to alter the drag polar - he can not, for example, eliminate the fuselage, transonic drag rise, or induced drag. Consistent with the rubber airplane assumption, the drag polar is held constant for this study.

As seen in Figures 3, the wing loading is a direct means for the designer to change the cruise aerodynamic efficiency of an aircraft. This influence stems from the role of the wing loading in determining the cruise lift coefficient and the role of the lift coefficient in determining where on the drag polar the aircraft will operate. Equation 4 for the cruise lift coefficient clearly shows the influence of wing loading, cruise weight fraction, and dynamic pressure $(q)$.

$$
C_{L}=\frac{\beta}{q} \frac{W_{T O}}{S}
$$

The wing loading and flight condition (through dynamic pressure) determine the lift coefficient and thereby the operating point on the drag polar. Through the wing loading, the designer can effectively influence the cruise aerodynamic efficiency at a specified flight condition.

\section{Propulsive Efficiency}

The effect of propulsive efficiency on aircraft range can be understood directly through TSFC. Figure 2(b) depicts typical behavior of $T S F C$ throughout the flight envelope. TSFC improves with increasing altitude until the tropopause and is constant thereafter; it generally degrades with increasing Mach number.

The engine designer, through selection of a thermodynamic cycle and its parameters has the greatest influence over this bulk behavior of $T S F C$. The aircraft designer typically chooses between existing engines or works with an engine manufacturer to develop an engine for an application. Consistent with the rubber engine assumption, $T / W$ is used by the conceptual aircraft designer to scale a given engine cycle to the desired aircraft application.

The effect of $T / W$ on the cruise efficiency of an aircraft is best understood by comparing the thrust hooks depicted in Figure 4. These hooks represent the same engine cycle scaled to three $T / W$ ratios each capable of sustaining flight $(T \geq D)$ at the specified flight condition. The first case, labeled $83 \%$, represents an under-sized engine where the engine must operate at nearly full power during cruise; this incurs a TSFC penalty. The second case, labeled $100 \%$, represents an engine sized such that cruise operation occurs at minimum TSFC. The final case, labeled $150 \%$, represents an over-sized engine where the engine must operate at greatly reduced power during cruise; operation near flight idle incurs a TSFC penalty as well as an implied engine weight penalty.

\section{Range Parameter as Point Performance}

The range parameter has been identified as a point performance metric indicative of the range efficiency of an aircraft and which is sensitive to changes in $T / W$ and $W / S$. As such, it will be appropriate to overlay a traditional constraint diagram with contours of range parameter. This calculation requires determination of the throttle setting for cruise flight.

First, recall that the thrust ratio for a rubberized engine is the thrust at a particular operating condition divided by the rated thrust of the engine i.e. $\alpha \equiv T / T_{s l s, \text { max }}$. This ratio includes thrust changes due to 


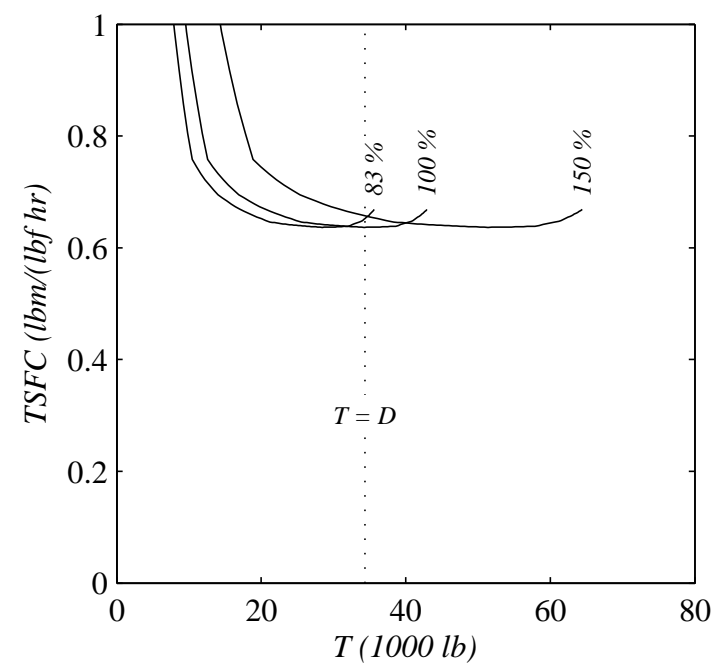

Figure 4. Effect of over/under sizing engine for cruise.

lapse and throttling. In cruise, thrust must equal drag. This condition is used to identify the cruise required thrust fraction, $\alpha_{r e q}$, as specified in Equation 5.

$$
\alpha_{r e q}=\frac{D}{T_{s l s, \max }}
$$

The definition of the drag coefficient is used to substitute the drag in this equation. Then the numerator and denominator are each multiplied by the takeoff gross weight, $W_{T O}$, resulting in the following expression.

$$
\alpha_{r e q}=\frac{C_{D} q S}{T_{s l s, \max }} \quad \frac{W_{T O}}{W_{T O}}
$$

This expression can be rearranged to be in terms of $W / S$ and $T / W$. Equation 6 gives the required cruise thrust fraction for operation at a given flight condition. An arbitrary engine deck will give engine performance as a function of flight condition and power code $P C$, i.e. $(\alpha, T S F C)=f(M, h, P C)$. In this case, the power code required to match $\alpha=\alpha_{r e q}$ can be solved for numerically.

$$
\alpha_{r e q}=C_{D} q \frac{S}{W_{T O}} \quad \frac{T_{s l s, \max }}{W_{T O}}
$$

Evaluation of Equation 6 also requires evaluation of $C_{D}$. An arbitrary drag polar will give drag coefficient as a function of flight condition and lift coefficient, i.e. $C_{D}=f\left(C_{L}, M, h\right)$. As shown in Equation 4, lift coefficient is also a function of wing loading.

With lift and drag coefficients known, the numerical solution of the cruise power code is possible. This calculation will also yield the cruise fuel consumption, TSFC. Consequently, all terms required for the calculation of range parameter via Equation 3 are known.

Contours of range parameter on axes of $T / W$ vs. $W / S$ for the example transport aircraft were generated and plotted as Figure 5. The cruise condition is $M=0.8$ at $h=36,000 \mathrm{ft}$. The zero excess power constraint curve for that flight condition is also included.

In terms of range parameter, the optimum $T / W$ is approximately 0.21 and the optimum $W / S$ is approximately 131lbf $/ \mathrm{ft}^{2}$. Changing $W / S$ results in moving along the drag polar to different values of $L / D$ at cruise. The optimum cruise point is located directly above the minimum $T / W$ point in the $P_{s}=0$ constraint curve. This minimum occurs at minimum drag and therefore best $L / D$. This heuristic can be used to identify the optimum cruise point in lieu of the complete range parameter calculation.

Changing $T / W$ results in moving along the thrust hook for the engine with accompanying changes in $T S F C$ as depicted in Figure4. If $T / W$ is increased greatly, the aircraft cruises near flight idle with a 


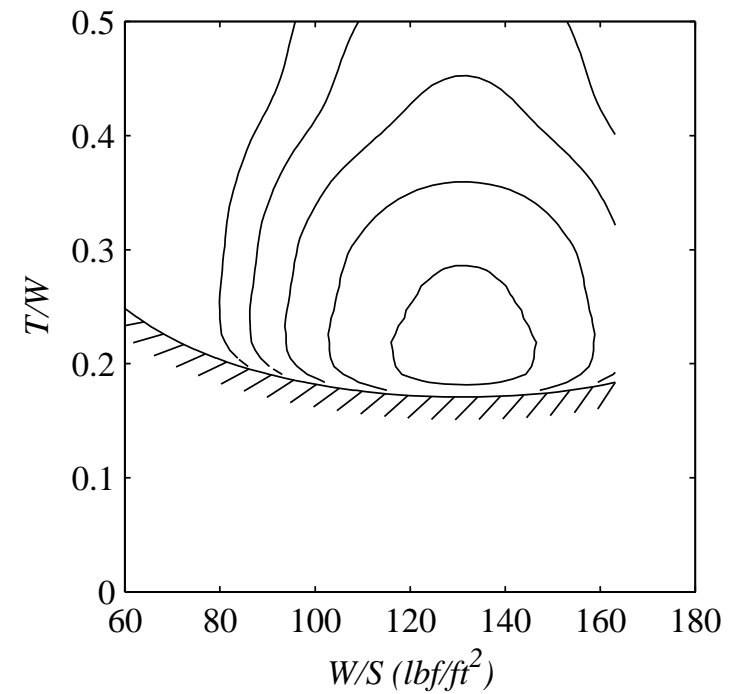

Figure 5. Example $T / W W / S$ constraint diagram depicting range parameter contours.

significant penalty in $T S F C$. If $T / W$ is decreased to the $P_{s}=0$ contour, the aircraft cruises at maximum throttle. In this case, full throttle operation comes with a slight TSFC penalty.

If, early in the design process, a complete thrust hook is unavailable, then the contours of range parameter will be vertical lines which only depict the impact of $L / D$ changes due to $W / S$. However, if the thrust fraction (say $80 \%$ ) for best $T S F C$ is known, then the optimum $T / W$ can be found by raising the minimum point in the $P_{s}=0$ constraint curve by dividing by this fraction.

These two heuristics can be used to identify the range parameter optimum $T / W$ and $W / S$ based on the cruise $P_{s}=0$ constraint curve. Although this shortcut accurately identifies the location of the optimum, it does not give any indication of the penalty associated with any non-optimal point.

\section{Conclusions}

The traditional $T / W$ vs. $W / S$ constraint diagram has been extended to include a measure of range efficiency - not as a constraint, but as contours of merit. These range parameter contours are developed using 'rubber engine' and 'rubber airplane' assumptions consistent with those used in the generation of a traditional constraint diagram. Generation of these contours has been demonstrated for an example aircraft based on complex, real-world aerodynamic and engine performance models.

The additional insight provided by this extension provides the designer with an immediate means to better understand the fundamental tradeoff between an aircraft's point performance and mission performance. This approach has been used with good success in recent years by the author's design students to help them make better design decisions while learning the aircraft design process.

\section{References}

\footnotetext{
${ }^{1}$ Mattingly, J. D., Aircraft Engine Design, AIAA, 2002.

${ }^{2}$ Raymer, D. P., Aircraft Design: A Conceptual Approach, AIAA, 2006.

${ }^{3}$ Roskam, J., Airplane Design, Part I: Preliminary Sizing of Airplanes, DAR Corporation, 2003.

${ }^{4}$ Torenbeek, E., "The Initial Calculation of Range and Mission Fuel During Conceptual Design," Tech. Rep. LR-525, Delft University of Technology, August 1987.

${ }^{5}$ McDonnell Douglas, Performance Course, 1978.

${ }^{6}$ General Electric, The CF6 Engine Installation Manual, March 31973.
} 